\title{
Belgeo
}

Revue belge de géographie

1-2| 2006

Competition and complementarity in retailing

\section{Editorial : Commerce de détail, concurrence et géographie}

Jean-Pierre Grimmeau

\section{(2) OpenEdition}

1 Journals

Édition électronique

URL : http://journals.openedition.org/belgeo/10850

DOI : 10.4000/belgeo.10850

ISSN : 2294-9135

Éditeur :

National Committee of Geography of Belgium, Société Royale Belge de Géographie

Édition imprimée

Date de publication : 30 juin 2006

Pagination : 1-8

ISSN : 1377-2368

Référence électronique

Jean-Pierre Grimmeau, «Editorial : Commerce de détail, concurrence et géographie », Belgeo [En ligne],

1-2 | 2006, mis en ligne le 18 décembre 2013, consulté le 22 septembre 2020. URL : http://

journals.openedition.org/belgeo/10850; DOI : https://doi.org/10.4000/belgeo.10850

Ce document a été généré automatiquement le 22 septembre 2020.

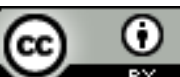

Belgeo est mis à disposition selon les termes de la licence Creative Commons Attribution 4.0 International. 


\title{
Editorial : Commerce de détail, concurrence et géographie
}

\author{
Jean-Pierre Grimmeau
}

1 Ce numéro porte à la fois sur les concurrences et sur les complémentarités dans le commerce de détail en Europe. Par concurrence on n'entend pas seulement la concurrence individuelle entre magasins mais aussi les concurrences plus générales entre formes de commerce, entre espaces ou entre clientèles différents. Le thème de la complémentarité est indissociable : certains commerces de même type ou s'adressant à un même type de clientèle tendent à se regrouper. La priorité a été donnée aux études empiriques, ayant documenté factuellement les concurrences et/ou complémentarités. La volonté a été que les études de cas dépassent la monographie locale en replaçant les phénomènes analysés localement dans une perspective plus large par la mise en évidence de mécanismes devant jouer aussi ailleurs. Les aspects historiques n'ont pas été écartés, mais limités à la seconde moitié du XX ${ }^{\mathrm{e}}$ siècle.

2 Le premier thème abordé est celui de la concurrence ou de la complémentarité entre le commerce moderne et le commerce urbain traditionnel. C'est évidemment en exEurope de l'est que l'évolution est la plus récente et la plus spectaculaire. Pommois retrace les transformations du commerce à Prague depuis le régime communiste ainsi que les conséquences sur l'évolution urbaine et les comportements d'achat. Dans ces pays, l'expansion du grand commerce n'est pas encore régulée par l'Etat, ce qui peut mener au suréquipement. Therin (2006) relève par exemple: «Varsovie abrite 23 hypermarchés de plus de $10000 \mathrm{~m}^{2}$ et son cinquième centre commercial géant devrait ouvrir ses portes en plein centre-ville en 2006. Lyon, qui possède une population comparable mais aux revenus bien plus confortables, ne compte, elle, que 10 hypermarchés et un mall». Kulke montre que, en ex-RDA, le développement du commerce moderne ne s'est trouvé sous contrôle de la législation allemande d'aménagement du territoire qu'au milieu des années 90 , laissant une brèche dans laquelle les investisseurs se sont rués (voir aussi la carte des centres commerciaux dans Institut für Länderkunde, 2002, p. 145). En Europe méridionale, le développement du commerce moderne est un peu plus ancien et maintenant généralement contrôlé. 
Carreras présente l'exemple intéressant de Barcelone, qui exporte maintenant son know-how. Loda parle entre autres de l'effet de l'arrivée des enseignes internationales sur le commerce à Florence. L'évolution de l'Allemagne présentée par Kulke est assez représentative, en tout cas pour la partie occidentale, des pays d'Europe du nord-ouest. Il présente entre autres des courbes d'évolution de la concentration et une réflexion sur les différences de localisations des différents types de commerces modernes. L'article de Hatz évoque aussi l'impact des enseignes internationales sur le commerce existant à Vienne. Borgers, van Swaaij \& Janssen proposent un modèle destiné à estimer l'impact de projets de centres commerciaux sur le commerce existant.

Dans la perspective d'augmenter l'attractivité des nouveaux projets, les investisseurs tablent sur le fun shopping (évoqué par Pommois et Carreras). C'est oublier un peu vite que le run shopping est une invention récente, particulièrement concrétisée par les hypermarchés. Au XIX ${ }^{e}$ siècle, les grands magasins et les galeries étaient fun, même s'ils étaient réservés aux happy few. C'est ce que rappelle Péron (2004, planches II et III) en mettant en parallèle deux gravures du XIX ${ }^{\mathrm{e}}$ siècle représentant l'une le hall d'un théâtre, l'autre l'intérieur d'un grand magasin. De même, Grunenberg (2002) présente Shopping as entertainment (pp. 21-22) mais c'est du XIX et de l'entre-deux-guerres qu'il parle!

second thème du numéro est la concurrence entre commerces de même type. Wayens et Grimmeau déterminent statistiquement pour Bruxelles quels types de commerces, selon une nomenclature fine, ont tendance à se repousser (concurrence classique) et lesquels ont tendance à se grouper (complémentarité). Ils examinent empiriquement les facteurs évoqués dans la littérature et concluent que, à l'exception du cas des banques, c'est la nécessité de comparaison qui pousse les commerces à se grouper. Léo et Philippe examinent la concurrence entre enseignes de restauration à Aix, Avignon et Marseille au moyen de la méthode de l'ensemble de considération. Ils étudient aussi l'évolution des stratégies de localisations de ces enseignes sur les dernières décennies. Sur le même thème, voir aussi Liarte (2004).

5 Le troisième thème du numéro est la concurrence entre types de clientèles et entre espaces à l'intérieur de la ville. Dans les villes à fort attrait touristique, un certain nombre de rues du centre-ville, les plus fréquentées par les touristes, se spécialisent vers cette clientèle (voir Grimmeau \& Wayens, 2006). En conséquence, les commerces destinés à la clientèle résidente deviennent plus rares, ce qui encourage encore la dépopulation du centre-ville. Cette question est ici particulièrement étudiée par Loda dans le cadre de Florence. Il montre aussi que les commerces destinés aux résidents, alimentaires et autres, deviennent moins rentables et sont de plus en plus tenus par des étrangers, généralement surqualifiés, qui trouvent ainsi une source de revenu alors que des raisons administratives les empêchent d'exercer une profession en accord avec leur formation. Cette surqualification s'accompagne généralement de l'absence de toute expérience ou qualification pour le commerce de détail, ce qui fragilise encore davantage ces commerces, dont Loda défend le soutien. La concurrence entre commerces pour touristes et pour résidents et les différentes mesures prises par les autorités locales sont aussi évoquées par Pommois, Carreras et Hatz. Un autre type de conflit possible entre types de clientèles concerne les commerces branchés. C'est le sujet de Van Criekingen et Fleury, qui examinent deux quartiers, l'un à Bruxelles, l'autre à Paris. Les différents commerces de ces espaces sont conscients de leur complémentarité. La concurrence ne se pose guère en terme commercial lors de la mise 
en place des commerces branchés, qui se fait dans des espaces en déprise, mais bien une fois l'orientation du quartier bien établie: Van Criekingen (1996 pp. 73-74) rapporte le doublement des loyers à Dansaert de 1985 à 1995. Dans le présent article, les auteurs rapportent l'arrivée d'enseignes dans cette même rue et la massification de la consommation à Oberkampf. Ces processus condamnent les commerces branchés à migrer vers les marges à mesure qu'ils relancent le succès des quartiers qu'ils investissent. De tels mécanismes jouent de façon plus générale pour expliquer les spécialisations progressives des différents quartiers commerçants du centre-ville, que Hatz met en évidence pour Vienne. Liarte (2004) suggère que les enseignes en quête de notoriété s'établissent près d'enseignes connues et que ce sont ces dernières qui peuvent innover en terme de localisation. Par exemple, l'irruption d'enseignes populaires dans une rue commerçante qui n'avait pas ce caractère peut modifier complètement les flux et les loyers ; van Haare Heijmeijer $(2002$, p. 10) cite un exemple à Louvain. Par ailleurs, la prolongation nocturne de l'activité commerciale peut engendrer des concurrences avec la fonction résidentielle (Loew, 2004).

Le dernier thème concerne les concurrences et complémentarités entre commerces dans les régions frontalières. Les différences de taxation entre pays modifient leur attrait relatif pour certains types de dépenses, que ce soit pour les frontaliers, les touristes ou les flux de passage. Le cas d'Andorre est à ce sujet exemplaire (Sanguin, 1991). En Belgique, on connaît mieux le cas du Grand-Duché de Luxembourg, où la taxation sur l'essence, l'alcool et le tabac est particulièrement réduite. La taxation sensiblement plus faible des eaux de boissons en France qu'en Belgique induit des flux importants, qui sont une aubaine pour les hypermarchés français (Patesson \& Steinberg, 2001). Hamez rapporte le cas du tabac, beaucoup moins taxé en Belgique qu'en France et au Royaume-Uni, au point de provoquer des flux doublement transfrontaliers, de l'Angleterre via la France vers une concentration de marchands de tabac apparus pour la circonstance en Belgique, juste au-delà de la frontière. Nous trouvons qu'il serait opportun d'étudier plus systématiquement les sur- ou sousreprésentations de types de commerces dans les zones frontalières, de façon à dresser un tableau plus complet des concurrences transfrontalières et de leurs facteurs. C'est ce que nous avons tenté pour la Belgique (tableau 1). Les zones frontalières sont définies sur une profondeur de $20 \mathrm{~km}$. Les indices représentent le rapport entre l'emploi observé et attendu sur base de la population et de la hiérarchie urbaine; les tests statistiques portent sur la différence entre ces effectifs (voir les détails de la méthodologie dans Wayens \& Grimmeau, 2006). Pour la plupart des types de commerces, les écarts sont significatifs. Le tableau reprend les plus significatifs. Même si l'analyse doit être approfondie, il est clair que les facteurs dépassent les seuls différentiels de taxation. D'autres éléments de la formation des prix interviennent. Les différences de législation sur le grand commerce interfèrent également (voir Borges, van Swaaij \& Janssen pour les spécificités des Pays-Bas et Jehin, 2002 pour une comparaison Belgique, Allemagne, Pays-Bas). Reynhardt (2006) défend par exemple l'idée que le moratoire interdisant la construction d'équipements commerciaux de plus de $10000 \mathrm{~m}^{2}$ au Grand-Duché de 1997 à 2005 est responsable de développements commerciaux en Belgique et en France près de la frontière. Smits montre, à propos de la concurrence commerciale entre la France, la Belgique, le Luxembourg et l'Allemagne dans le cadre de la Grande région, que les facteurs sont encore plus diversifiés. Interviennent par exemple les flux de travailleurs frontaliers, l'organisation régulière de marchés, la présence unilatérale d'enseignes ou de marques, les différences de standings, les heures et jours d'ouverture, etc. Outre la 
différence de prix, le facteur touristique n'est pas négligeable: faire du shopping à l'étranger c'est se dépayser, comme le faisait déjà remarquer Verhasselt (1966, pp. 278-284 et 351-355) à propos de l'attrait des villes hollandaises de Maastricht et de Sluis pour les Belges.

Tableau 1. Sur- et sous-représentations significatives de types de commerce dans les zones frontalières de la Belgique. Les indices sont le rapport entre l'emploi observé et l'emploi théorique tenant compte de la population et de la hiérarchie urbaine. C'est la différence entre ces effectifs qui fait l'objet du test statistique.

\begin{tabular}{|c|c|c|c|c|c|c|c|c|}
\hline \multirow{2}{*}{ Tyal hermors } & \multicolumn{8}{|c|}{ 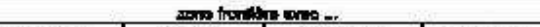 } \\
\hline & \multicolumn{2}{|c|}{ Alminger } & \multicolumn{2}{|c|}{ Fines } & \multicolumn{2}{|c|}{ Lussmbour } & \multicolumn{2}{|l|}{ Paw } \\
\hline spemberche & 74 & $\%$ & 7 & . & 91 & & 81 & \% \\
\hline hypemmarchest grands meguairs & 2 & E & $s$ & & 278 & - & $\pi$ & F \\
\hline sqence de whase & 40 & 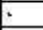 & 58 & $\cdot$ & 45 & - & $\infty$ &. \\
\hline dimertation genierde & 94 & & 139 & - & $\approx$ & & 99 & \\
\hline metartion & 91 & & 24 & $=$ & 73 & - & 110 & 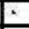 \\
\hline risnde & 120 & 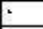 & 12 & $\cdot$ & 105 & & 121 & . \\
\hline meutle & 153 & - & $\infty$ & $\cdot$ & 130 & - & 128 & $\dot{1}$ \\
\hline coticettors & 99 & & 117 & 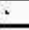 & 99 & & $1+3$ & - \\
\hline werrert gines al & 105 & & 81 & $=$ & 94 & & 118 & $\%$ \\
\hline Eoubragerie & 126 & - & 105 & & 112 & & 117 & . \\
\hline empldi & 98 & & 97 & $\cdot$ & 99 & & 100 & 5 \\
\hline metarationracide & 95 & L & 84 & 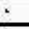 & 81 & & 109 & + \\
\hline contustitks & 167 & $E$ & 145 & 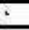 & 195 & - & 81 & 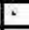 \\
\hline tabac & 141 & & 224 & - & 31 & & 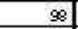 & \\
\hline squpemert du foves & 68 & - & $\approx$ & $\cdot$ & 99 & & $1+9$ & 1. \\
\hline entetien etreporation auto & 119 & - & 99 & & 130 & . & 100 & \\
\hline pompos funitres & 51 & - & s & & 56 & & $1+9$ & 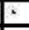 \\
\hline testie & 99 & & 108 & & 64 & & 117 & i \\
\hline whisules automatiles & 122 & - & 109 & $\cdot$ & 128 & - & 9 & \\
\hline guimaillesic & 87 & & 106 & & 169 & - & 97 & \\
\hline gokes & 100 & & $\pi$ & & 4 & & 148 & 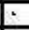 \\
\hline stonstarwirs tlematisseries: & 49 & & 132 & 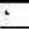 & 11 & - & $\pi$ & - \\
\hline slons de coiftires & 114 & - & 105 & & 107 & & 107 & . \\
\hline buresuordinzter & 62 & - & 81 & $\div$ & 78 & & 91 & \\
\hline peisson & 66 & & 144 & $\div$ & 4 & & 110 & \\
\hline phamracie & 117 & & 107 & & 100 & & 94 & 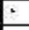 \\
\hline simaux & 69 & & 12 & $\cdot$ & 50 & & 10 & \\
\hline perimerie & 85 & & 90 & & 74 & & 118 &. \\
\hline themert enfornt & 74 & & $\pi$ & 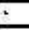 & 154 & & 90 & \\
\hline sosturarts & 124 & & 100 & & 3 & 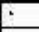 & 105 & \\
\hline antiquitt-brocente & 115 & & 76 & - & 70 & & $\approx$ & \\
\hline diractiogues & 113 & & 109 & & 77 & & 117 & - \\
\hline & & & & & & & & \\
\hline
\end{tabular}

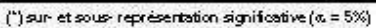

\section{BIBLIOGRAPHIE}

GRIMMEAU J.P. \& WAYENS B. (2006), « Tourisme et commerce à Bruxelles », Cahiers de la Fonderie, 34.

GRUNENBERG C. (2002), «Wonderland: spectacles of display from the Bon Marché to Prada ", in GRUNENBERG C. \& HOLLEIN M. (eds.), Shopping. A century of art and consummer culture, Hatje Cantz Publishers, pp. 17-37.

INSTITUT FÜR LÄNDERKUNDE (2002), Nationalatlas Bundesrepublik Deutschland, 5, Dörfer und Städte, Spektrum Akademischer Verlag. 
JEHIN J.B. (2002), «Influence des politiques des pouvoirs publics en matière d'implantations commerciales sur l'activité en centre-ville. Comparaison : Liège, Maastricht et Aix-la-Chapelle », Bulletin de la Société géographique de Liège, 42, pp. 39-51.

LIARTE S. (2004), « La proximité géographique comme stratégie concurrentielle face au leader : le cas des chaînes de restauration rapide à Paris entre 1984 et 2002 ", $13^{e}$ conférence de l'Association internationale de management stratégique, Normandie.

http://www.strategie-aims.com/Normandie04/sessions/Liarte.pdf

LOEW S. (2004), « La ville 24 h sur 24 en Angleterre », in BONDUE J.P. (éd.) Temps des courses, course du temps, Université de Lille 1, pp. 79-85.

PATESSON R. \& STEINBERG P. (2001), Les emballages pour boissons : enquête auprès des ménages belges et des acheteurs transfrontaliers, Rapport de synthèse du 23 avril 2001, Centre de psychosociologie de l'opinion, $15 \mathrm{p}$.

http://www.ulb.ac.be/soco/creatic/emballages.pdf

PERRON R. (2004), Les boîtes. Les grandes surfaces dans la ville, L'Atalante.

REYNHARDT A. (2006), «Le Luxembourg donne de l'air à son immobilier commercial », LSA, $n^{\circ} 1951$, p. 27.

SANGUIN A.-L. (1991,) «L'Andorre ou la quintessence d'une économie transfrontalière », Revue géographique des Pyrénées et du Sud-Ouest, 62-2, pp. 169-186.

THERIN F. (2006), « L'Etat polonais inquiète les distributeurs », LSA, nº1935, pp. 26-28.

VAN CRIEKINGEN M. (1996), Processus de gentrification à Bruxelles : le cas du quartier « Dansaert SaintGéry", mémoire de licence en géographie, Université libre de Bruxelles.

VAN HAARE HEIJMEIJER B. (2002), « Marché de l'immobilier commercial en Belgique : quelles perspectives ? ", Urbanisme commercial, 20-21 mars, IFE, Bruxelles.

VERHASSELT Y. (1966), Les frontières du nord et de l'est de la Belgique, Société royale belge de géographie.

WAYENS B. \& GRIMMEAU J.P. (2006) «SIG et analyse des localisations commerciales », in Savoir penser et partager l'information géographique : les SIG, Géoforum 2005, Association française pour le développement de la géographie, pp. 77-83.

\section{AUTEUR}

\section{JEAN-PIERRE GRIMMEAU}

Institut de Gestion de l'Environnement et d'Aménagement du Territoire, Université Libre de Bruxelles, grimmeau@ulb.ac.be 